ALEA, Lat. Am. J. Probab. Math. Stat. 15, 1141-1161 (2018)

DOI: $10.30757 /$ ALEA.v15-42

\title{
Stein normal approximation \\ for multidimensional Poisson random measures by third cumulant expansions
}

\author{
Nicolas Privault \\ Division of Mathematical Sciences \\ School of Physical and Mathematical Sciences \\ Nanyang Technological University \\ 21 Nanyang Link, Singapore 637371. \\ E-mail address: nprivault@ntu.edu.sg \\ URL: http://www.ntu.edu.sg/home/nprivault/
}

\begin{abstract}
We derive normal approximation bounds by the Stein method for stochastic integrals with respect to a Poisson random measure over $\mathbb{R}^{d}, d \geq 2$. This approach relies on third cumulant Edgeworth-type expansions based on derivation operators defined by the Malliavin calculus for Poisson random measures. The use of third cumulants can exhibit faster convergence rates than the standard BerryEsseen rate for some sequences of Poisson stochastic integrals.
\end{abstract}

\section{Introduction}

Normal approximation bounds for stochastic integrals with respect to a Poisson random measure have been obtained by the Stein method in Peccati et al. (2010), using finite difference operators on the Poisson space. Recent results in this direction include the proof of a fourth moment theorem (see Döbler and Peccati, 2018 and Döbler et al., 2018), as an extension of the result of Nualart and Peccati (2005) to the setting of Poisson point processes.

In this paper we derive related bounds for compensated Poisson stochastic integrals $\delta(u):=\int_{\mathbb{R}^{d}} u_{x}(\gamma(d x)-\lambda(d x))$ of processes $\left(u_{x}\right)_{x \in \mathbb{R}^{d}}$ with compact support in $\mathbb{R}^{d}$, with respect to a Poisson random measure $\gamma(d x)$ with intensity the Lebesgue measure $\lambda(d x)$ on $\mathbb{R}^{d}, d \geq 2$. In contrast with Peccati et al. (2010), our approach is based on derivation operators and Edgeworth-type expansions that involve the

Received by the editors November 12th, 2017; accepted September 10th, 2018.

2010 Mathematics Subject Classification. 62E17, 60H07, 60H05.

Key words and phrases. Stein approximation; multidimensional Poisson random measures; Poisson stochastic integrals; cumulants; Malliavin calculus; Edgeworth expansions.

Research supported by Singapore MOE Tier 2 Grant MOE2016-T2-1-036. 
third cumulant of Poisson stochastic integrals, and can result into faster convergence rates, see e.g. (1.5) below.

Edgeworth-type expansions have been obtained on the Wiener space in Nourdin and Peccati (2009a), Campese (2013), by a construction of cumulant operators based on the inverse $L^{-1}$ of the Ornstein-Uhlenbeck operator, extending the results of Nourdin and Peccati (2009b) on Stein approximation and Berry-Esseen bounds.

In Proposition 4.1 we derive Edgeworth-type expansions of the form

$$
\begin{aligned}
\mathbb{E}[\delta(u) g(\delta(u))]= & \mathbb{E}\left[\|u\|_{L^{2}\left(\mathbb{R}^{d}\right)}^{2} g^{\prime}(\delta(u))\right] \\
& +\sum_{k=2}^{n} \mathbb{E}\left[g^{(k)}(\delta(u)) \Gamma_{k+1}^{u} \mathbf{1}\right]+\mathbb{E}\left[g^{(n+1)}(\delta(u)) R_{n}^{u}\right]
\end{aligned}
$$

when the random field $\left(u_{x}\right)_{x \in \mathbb{R}^{d}}$ is predictable with respect to a given total order on $\mathbb{R}^{d}$, where $\Gamma_{k}^{u}$ is a cumulant-type operator and $R_{n}^{u}$ is a remainder term, defined using the derivation operators of the Malliavin calculus on the Poisson space. In comparison with the results of Peccati et al. (2010), our bounds apply to a different stochastic integral representation of random variables, and they allow for random integrands $\left(u_{x}\right)_{x \in \mathbb{R}^{d}}$. In particular, this allows us to deal with random variables $\delta(u)$ having infinite chaos expansions.

Based on (1.1), in Corollary 5.1 we deduce Stein approximation bounds of the form

$$
\begin{aligned}
d_{W}(\delta(u), \mathcal{N}) \leq & |1-\operatorname{Var}[\delta(u)]|+\sqrt{\operatorname{Var}\left[\|u\|_{L^{2}\left(\mathbb{R}^{d}\right)}^{2}\right]} \\
& +\mathbb{E}\left[\left|\int_{\mathbb{R}^{d}} u_{x}^{3} \lambda(d x)+\left\langle u, D \int_{\mathbb{R}^{d}} u_{x}^{2} \lambda(d x)\right\rangle_{L^{2}\left(\mathbb{R}^{d}\right)}\right|\right]+\mathbb{E}\left[\left|R_{1}^{u}\right|\right],
\end{aligned}
$$

where $D$ is a gradient operator acting on Poisson functionals, and $\mathcal{N} \simeq \mathcal{N}(0,1)$ is a standard Gaussian random variable, see also Proposition 5.1. Here,

$$
d_{W}(F, G):=\sup _{h \in \mathcal{L}}|\mathbb{E}[h(F)]-\mathbb{E}[h(G)]|
$$

is the Wasserstein distance between the laws of two random variables $F$ and $G$, where $\mathcal{L}$ denotes the class of 1-Lipschitz functions on $\mathbb{R}$.

In particular, when $f$ is a differentiable deterministic function on the closed centered ball $B(R):=B(0 ; R)$ in $\mathbb{R}^{d}$ with radius $R>0$, vanishing on the sphere $S(0 ; R):=\left\{x \in \mathbb{R}^{d}:|x|=R\right\}$, we obtain bounds of the form

$$
\begin{aligned}
d_{W}\left(\int_{\mathbb{R}^{d}} f(x)(\gamma(d x)-\lambda(d x)), \mathcal{N}\right) \leq & \left|1-\|f\|_{L^{2}\left(\mathbb{R}^{d}\right)}^{2}\right|+\left|\int_{\mathbb{R}^{d}} f^{3}(x) \lambda(d x)\right|(1.2) \\
& +8\left(K_{d} v_{d} R\right)^{2}\|f\|_{L^{2}\left(\mathbb{R}^{d}\right)}\left\|\nabla^{\mathbb{R}^{d}} f\right\|_{L^{\infty}\left(\mathbb{R}^{d} ; \mathbb{R}^{d}\right)}^{2},
\end{aligned}
$$

where $v_{d}$ denotes the volume of the unit ball in $\mathbb{R}^{d}$ and $K_{d}>0$ is a constant depending only on $d \geq 2$. The bound (1.2) can be compared to the classical Stein bound

$$
d_{W}\left(\int_{\mathbb{R}^{d}} f(x)(\gamma(d x)-\lambda(d x)), \mathcal{N}\right) \leq\left|1-\|f\|_{L^{2}\left(\mathbb{R}^{d}\right)}^{2}\right|+\int_{\mathbb{R}^{d}}\left|f^{3}(x)\right| \lambda(d x),
$$


for compensated Poisson stochastic integrals, see Corollary 3.4 of Peccati et al. (2010), which involves the $L^{3}\left(\mathbb{R}^{d}\right)$ norm of $f$ instead of third cumulant $\kappa_{3}^{f}=$ $\int_{\mathbb{R}^{d}} f^{3}(x) \lambda(d x)$ of $\int_{\mathbb{R}^{d}} f(x)(\gamma(d x)-\lambda(d x))$, and relies on the use of finite difference operators, see Theorem 3.1 of Peccati et al. (2010) and $\S 4.2$ of Bourguin and Peccati (2016).

For example when $f_{k}, k \geq 1$, is a radial function given on $B\left(k^{1 / d} R\right)$ by

$$
f_{k}(x):=\frac{1}{C \sqrt{k}} g\left(\frac{|x|_{\mathbb{R}^{d}}}{k^{1 / d}}\right), \quad x \in B\left(k^{1 / d} R\right),
$$

where $g \in \mathcal{C}^{1}([0, R])$ is continuously differentiable on $[0, R]$ with $g(R)=0$, and

$$
C^{2}:=\int_{0}^{R} g^{2}(r) r^{d-1} d r<\infty
$$

so that $\left\|f_{k}\right\|_{L^{2}\left(B\left(k^{1 / d} R\right)\right)}=1$, the bound (1.3) yields the standard Berry-Esseen convergence rate

$$
d_{W}\left(\int_{B\left(k^{1 / d} R\right)} f_{k}(x)(\gamma(d x)-\lambda(d x)), \mathcal{N}\right) \leq \frac{v_{d}}{C^{3} \sqrt{k}} \int_{0}^{R}|g(r)|^{3} r^{d-1} d r, \quad k \geq 1,
$$

as $k$ tends to infinity. While (1.2) does not improve on (1.3) when the function $f$ has constant sign, if $g$ satisfies the condition

$$
\int_{0}^{R} g^{3}(r) r^{d-1} d r=0
$$

then the third cumulant bound (1.2) yields the $O(1 / k)$ convergence rate

$$
d_{W}\left(\int_{B\left(k^{1 / d} R\right)} f_{k}(x)(\gamma(d x)-\lambda(d x)), \mathcal{N}\right) \leq \frac{2\left(2 K_{d} v_{d} R\right)^{2} d}{k C^{2}}\left\|g^{\prime}\right\|_{\infty}^{2}, \quad k \geq 1
$$

which improves on the standard Berry-Esseen rate, see Section 5 for more examples.

In Sections 2 and 3 we recall some background material on the Malliavin calculus and differential geometry on the Poisson space, by revisiting the approach of Prat and Privault (1999), Privault (2001) using the recent constructions of Acosta and Durán (2017) and references therein on the solution of the divergence problem. In Section 4 we derive Edgeworth-type expansions for the compensated Poisson stochastic integral $\delta(u)$, based on a family of cumulant operators that are associated to the random field $\left(u_{x}\right)_{x \in \mathbb{R}^{d}}$. In Section 5 we obtain Stein-type approximation bounds for stochastic integrals using deterministic examples of integrands.

The $d$-dimensional setting of this paper requires $d \geq 2$ and a bounded domain in $\mathbb{R}^{d}$ in order to construct a gradient operator $D$ for Poisson functionals by kernel inversion of the divergence operator on $\mathbb{R}^{d}$ using results of Acosta and Durán (2017) and references therein. Consequently it does not cover the case $d=1$ of the standard Poisson process on the half line $\mathbb{R}_{+}$, which requires a significantly different treatment, see Privault $(2018+)$. In particular, the one-dimensional case is technically easier as it does not require Laplace inversion for the construction 
of the gradient operator $D$, while stronger conditions on the integrands $f$ in Poisson stochastic integrals have to be imposed in the case $d \geq 2$ through the norm $\left\|\nabla^{\mathbb{R}^{d}} f\right\|_{L^{\infty}\left(\mathbb{R}^{d} ; \mathbb{R}^{d}\right)}$.

Preliminaries. Let $d \geq 2$ and $0<R<R^{\prime}:=2 R$. We let $\mathcal{C}_{0}^{\infty}\left(B\left(R^{\prime}\right)\right)$ denote the space of $\mathcal{C}^{\infty}$ functions on $B\left(R^{\prime}\right)$ which vanish on the sphere $S\left(0 ; R^{\prime}\right)=\left\{x \in \mathbb{R}^{d}\right.$ : $\left.|x|=R^{\prime}\right\}$. Given $\eta \in \mathcal{C}_{0}^{\infty}\left(B\left(R^{\prime}\right)\right)$ such that $\int_{B(R)} \eta(x) d x=1$, we recall the existence of a $\mathcal{C}^{\infty}$ kernel function $\mathrm{G}_{\eta}: B\left(R^{\prime}\right) \times B\left(R^{\prime}\right) \rightarrow \mathbb{R}^{d}$ defined as

$$
\mathrm{G}_{\eta}(x, y):=\int_{0}^{1} \frac{(x-y)}{s} \eta\left(y+\frac{x-y}{s}\right) \frac{d s}{s^{d}}, \quad x, y \in B\left(R^{\prime}\right),
$$

see Acosta and Durán (2017), and satisfying the following properties:

i) The kernel $\mathrm{G}_{\eta}(x, y)$ satisfies the bound

$$
\left|\mathrm{G}_{\eta}(x, y)\right|_{\mathbb{R}^{d}} \leq \frac{K_{d}}{|x-y|_{\mathbb{R}^{d}}^{d-1}}, \quad x, y \in B\left(R^{\prime}\right),
$$

for a constant $K_{d}>0$ depending only on $d$, see Lemma 2.1 of Acosta and Durán (2017), by choosing $K_{d}$ and the function $\eta \in \mathcal{C}_{c}^{\infty}\left(B\left(R^{\prime}\right)\right)$ therein so that $\|\eta\|_{\infty} \leq(d-1) K_{d}\left(R^{\prime}\right)^{-d}$.

ii) For any $p>1$ and $g \in L^{p}\left(B\left(R^{\prime}\right)\right)$ the function

$$
f(x):=\int_{B\left(R^{\prime}\right)} \mathrm{G}_{\eta}(x, y) g(y) \lambda(d y), \quad x \in B\left(R^{\prime}\right),
$$

satisfies the bound

$$
\|f\|_{L^{p}\left(B\left(R^{\prime}\right) ; \mathbb{R}^{d}\right)} \leq K_{d} v_{d} R^{\prime}\|g\|_{L^{p}\left(B\left(R^{\prime}\right)\right)}, \quad p>1,
$$

which follows from Young's inequality and (1.6), cf. Theorem 2.4 in Acosta and Durán (2017).

iii) For any $h \in \mathcal{C}_{0}^{\infty}\left(B\left(R^{\prime}\right)\right)$ we have the relation

$h(y)-\int_{B\left(R^{\prime}\right) \backslash B(R)} h(x) \eta(x) \lambda(d x)=\int_{B\left(R^{\prime}\right)}\left\langle\mathrm{G}_{\eta}(x, y), \nabla_{x}^{\mathbb{R}^{d}} h(x)\right\rangle_{\mathbb{R}^{d}} \lambda(d x), y \in B\left(R^{\prime}\right)$,

cf. Lemma 2.2 in Acosta and Durán (2017), by taking $\eta \in \mathcal{C}_{c}^{\infty}\left(B\left(R^{\prime}\right) \backslash B(R)\right)$. In particular, when $h \in \mathcal{C}_{0}^{\infty}(B(R))$ we have

$$
h(y)=\int_{B\left(R^{\prime}\right)}\left\langle\mathrm{G}_{\eta}(x, y), \nabla_{x}^{\mathbb{R}^{d}} h(x)\right\rangle_{\mathbb{R}^{d}} \lambda(d x), \quad y \in B\left(R^{\prime}\right) .
$$

An extension of the framework of this paper, by replacing $B(R)$ with a compact $d$-dimensional Riemannian manifold $M$ and $\lambda(d x)$ with the volume element of $M$, would require the Laplacian $\mathcal{L}=\operatorname{div}^{M} \nabla^{M}$ to be invertible on $\mathcal{C}_{c}^{\infty}(M)$ with

$$
\mathcal{L}^{-1} u(x)=\int_{M} \mathrm{~g}(x, y) u(y) \lambda(d y), \quad x \in M, u \in \mathcal{C}_{c}^{\infty}(M),
$$

where $\mathrm{g}(x, y)$ is the heat kernel on $M$. In this case we can define $\mathrm{G}_{\eta}(x, y) \in \mathbb{R}^{d}$ as

$$
\mathrm{G}_{\eta}(x, y)=\nabla_{x}^{M} \mathrm{~g}(x, y), \quad \lambda \otimes \lambda(d x, d y)-\text { a.e. }
$$

with the relation

$$
\nabla_{x}^{M} \mathcal{L}^{-1} u(x)=\int_{M} u(y) \mathrm{G}_{\eta}(x, y) \lambda(d y) \in T_{x} M, \quad x \in M, u \in \mathcal{C}_{c}^{\infty}(M),
$$


from which the divergence inversion relation (1.9) holds by duality.

\section{Gradient, divergence and covariance derivative}

There exists different notions of gradient and divergence operators for functionals of Poisson random measures. The operators of Albeverio et al. (1998), Privault and Torrisi (2011), Clausel et al. (2016), and their associated integration by parts formula rely on an $\mathbb{R}^{d}$-valued gradient for random functionals and a divergence operator which is associated to the non-compensated Poisson stochastic integral of the divergence of $\mathbb{R}^{d}$-valued random fields. This particularity, together with a lack of a suitable commutation relation between gradient and divergence operators on Poisson functionals, makes this framework difficult to use for a direct analysis of Poisson stochastic integrals, while it has found applications to statistical estimation and sensitivity analysis, see Clausel et al. (2016), Privault and Torrisi (2011).

In this paper we use the construction of Prat and Privault (1999), Privault (2001) which relies on real-valued tangent processes and on a divergence operator that directly extends the compensated Poisson stochastic integral. This framework also allows for simple commutation relations between gradient and divergence operators using the deterministic inner product in $L^{2}\left(\mathbb{R}^{d}, \lambda\right)$, see Proposition 2.3, and it naturally involves the Poisson cumulants, see Definition 3.1 and Relation (3.6).

Gradient operator. In the sequel we consider a Poisson random measure $\gamma(d x)$ on $B(R)$, constructed on a probability space $(\Omega, \mathcal{F}, P)$, and we let $\left\{X_{1}, \ldots, X_{n}\right\}$ denote the configuration points of $\gamma(d x)$ when $B(R)$ contains $n$ points in the configuration $\gamma$, i.e. when $\gamma(B(R))=n$.

Definition 2.1. Given $A$ a closed subset of $B\left(R^{\prime}\right)$, we let $\mathcal{S}_{A}$ denote the set of random functionals $F_{A}$ of the form

$$
F_{A}=\sum_{n=0}^{\infty} \mathbf{1}_{\{\gamma(B(R))=n\}} f_{n}\left(X_{1}, \ldots, X_{n}\right),
$$

where $f_{0} \in \mathbb{R}$ and $\left(f_{n}\right)_{n \geq 1}$ is a sequence of functions satisfying the following conditions:

- for all $n \geq 1, f_{n} \in \mathcal{C}_{c}^{\infty}\left(A^{n}\right)$ is a symmetric function in $n$ variables,

- for all $n \geq 1$ and $i=1, \ldots, n$ we have the continuity condition

$$
f_{n}\left(x_{1}, \ldots, x_{n}\right)=f_{n-1}\left(x_{1}, \ldots, x_{i-1}, x_{i+1}, \ldots, x_{n}\right),
$$

for all $x_{1}, \ldots, x_{n} \in B\left(R^{\prime}\right)$ such that $\left|x_{i}\right|_{\mathbb{R}^{d}} \geq R$.

We also let $\mathcal{S}$ denote the union of the sets $\mathcal{S}_{A}$ over the closed subsets $A$ of $B\left(R^{\prime}\right)$.

The gradient operator $D$ is defined on random functionals $F \in \mathcal{S}$ of the form (2.1) as

$$
D_{y} F:=\sum_{n=1}^{\infty} \mathbf{1}_{\{\gamma(B(R))=n\}} \sum_{i=1}^{n}\left\langle\mathrm{G}_{\eta}\left(X_{i}, y\right), \nabla_{x_{i}}^{\mathbb{R}^{d}} f\left(X_{1}, \ldots, X_{n}\right)\right\rangle_{\mathbb{R}^{d}}
$$

$y \in B(R)$. For any $F \in \mathcal{S}$, by (1.6) we have $D F \in L^{1}(\Omega \times B(R))$ from the bound

$$
\mathbb{E}\left[\int_{B(R)}\left|D_{x} F\right| \lambda(d x)\right] \leq\left\|\left|\nabla^{\mathbb{R}^{d}} f\right|_{\mathbb{R}^{d}}\right\|_{\infty} \mathbb{E}\left[\int_{B(R)} \int_{B(R)}\left|\mathrm{G}_{\eta}(x, y)\right|_{\mathbb{R}^{d}} \gamma(d x) \lambda(d y)\right]
$$




$$
\begin{aligned}
& =\left\|\left|\nabla^{\mathbb{R}^{d}} f\right|_{\mathbb{R}^{d}}\right\|_{\infty} \int_{B(R)} \int_{B(R)}\left|G_{\eta}(x, y)\right|_{\mathbb{R}^{d}} \lambda(d x) \lambda(d y) \\
& =K_{d}\left\|\left|\nabla^{\mathbb{R}^{d}} f\right|_{\mathbb{R}^{d}}\right\|_{\infty} \int_{B(R)} \int_{B(R)} \frac{1}{|x-y|_{\mathbb{R}^{d}}^{d-1}} \lambda(d x) \lambda(d y) \\
& \leq K_{d} v_{d}^{2} R^{\prime} R^{d}\left\|\left|\nabla^{\mathbb{R}^{d}} f\right|_{\mathbb{R}^{d}}\right\|_{\infty} \\
& <\infty
\end{aligned}
$$

Poisson-Skorohod integral. We let $\mathcal{U}_{0}$ denote the space of simple random fields of the form

$$
u=\sum_{i=1}^{n} g_{i} G_{i}, \quad n \geq 1
$$

with $G_{i} \in \mathcal{S}_{A_{i}}$ and $g_{i} \in \mathcal{C}_{0}^{\infty}(B(R)), i=1, \ldots, n$.

Definition 2.2. We define the Poisson-Skorohod integral $\delta(u)$ of $u \in \mathcal{U}_{0}$ of the form (2.4) as

$$
\delta(u):=\sum_{i=1}^{n}\left(G_{i} \int_{B(R)} g_{i}(x)(\gamma(d x)-\lambda(d x))-\left\langle g_{i}, D G_{i}\right\rangle_{L^{2}(B(R))}\right) .
$$

In particular, for $h \in \mathcal{C}_{0}^{\infty}(B(R))$ we have

$$
\delta(h)=\int_{B(R)} h(x)(\gamma(d x)-\lambda(d x)) .
$$

The proof of the next proposition, cf. Proposition 8.5.1 in Prat and Privault (1999) and Proposition 5.1 in Privault (2001), is given in the appendix.

Proposition 2.1. The operators $D$ and $\delta$ satisfy the duality relation

$$
\mathbb{E}\left[\langle u, D F\rangle_{L^{2}(B(R))}\right]=\mathbb{E}[F \delta(u)], \quad F \in \mathcal{S}, \quad u \in \mathcal{U}_{0} .
$$

As a consequence of Proposition 2.1 and the denseness of $\mathcal{S}$ in $L^{1}(\Omega)$ and that of $\mathcal{U}_{0}$ in $L^{1}(\Omega \times B(R))$, the gradient operator $D$ is closable in the sense that if $\left(F_{n}\right)_{n \in \mathbb{N}} \subset \mathcal{S}$ tends to zero in $L^{2}(\Omega)$ and $\left(D F_{n}\right)_{n \in \mathbb{N}}$ converges to $U$ in $L^{1}(\Omega \times B(R))$, then $U=0$ a.e.. Similarly, the divergence operator $\delta$ is closable in the sense that if $\left(u_{n}\right)_{n \in \mathbb{N}} \subset \mathcal{U}_{0}$ tends to zero in $L^{2}(\Omega \times B(R))$ and $\left(\delta\left(u_{n}\right)\right)_{n \in \mathbb{N}}$ converges to $G$ in $L^{1}(\Omega)$, then $G=0$ a.s..

The gradient operator $D$ defines the Sobolev space $\mathbb{D}^{1,1}$ with the Sobolev norm

$$
\|F\|_{\mathbb{D}_{1,1}}:=\|F\|_{L^{2}(\Omega)}+\|D F\|_{L^{1}(\Omega \times B(R))}, \quad F \in \mathcal{S} .
$$

In the sequel we fix a total order $\preceq$ on $B(R)$ and consider the space $\mathcal{P}_{0} \subset \mathcal{U}_{0}$ of simple predictable random field of the form

$$
u:=\sum_{i=1}^{n} g_{i} F_{i}
$$

such that the supports of $g_{1}, \ldots, g_{n}$ satisfy

$$
\operatorname{Supp}\left(g_{i}\right) \preceq \cdots \preceq \operatorname{Supp}\left(g_{n}\right) \text { and } \quad F_{i} \in \mathcal{S}_{A_{i}},
$$

where Supp $\left(g_{1}\right) \cup \cdots \cup \operatorname{Supp}\left(g_{i-1}\right) \subset A_{i} \subset B\left(R^{\prime}\right)$ and $A_{i} \preceq \operatorname{Supp}\left(g_{i}\right), i=1, \ldots, n$. 
Such random fields are predictable in the sense of e.g. $\S 5$ of Last (2016) and references therein.

We will also assume that the order $\preceq$ is compatible with the kernel $G_{\eta}$ in the sense that

$$
\mathrm{G}_{\eta}(x, y)=0 \quad \text { for all } \quad x, y \in B(R) \text { such that } x \preceq y .
$$

Under the compatibility condition (2.8) we have in particular

$$
D_{y} F=0, \quad y \in B(R), \quad A \preceq y, \quad F \in \mathcal{S}_{A} .
$$

Moreover, if $u \in \mathcal{P}_{0}$ is a predictable random field of the form (2.7) we note that by (2.3) and the compatibility condition (2.8) we have

$$
D_{y} F_{i}=0, \quad A_{i} \preceq y, \quad i=1, \ldots, n,
$$

hence

$$
D_{y} u_{x}=0, \quad x \preceq y, \quad x, y \in B(R) .
$$

Example. The order $\preceq$ defined by

$$
x=\left(x^{(1)}, \ldots, x^{(d)}\right) \preceq y=\left(y^{(1)}, \ldots, y^{(d)}\right) \quad \Longleftrightarrow \quad x^{(1)} \leq y^{(1)}
$$

is compatible with the kernel $\mathrm{G}_{\eta}$ provided that the support of $\eta$ is contained in

$$
\left\{x=\left(x^{(1)}, \ldots, x^{(d)}\right) \in B\left(R^{\prime}\right) \backslash B(R): x^{(1)}>R\right\} .
$$

The proof of the next Proposition 2.2 is given in the appendix.

Proposition 2.2. The Poisson-Skorohod integral of $u=\left(u_{x}\right)_{x \in B(R)}$ in the space $\mathcal{P}_{0}$ of simple predictable random fields satisfies the relation

$$
\delta(u)=\int_{B(R)} u_{x}(\gamma(d x)-\lambda(d x)),
$$

which extends to the closure of $\mathcal{P}_{0}$ in $L^{2}(\Omega \times B(R))$ by density and the isometry relation

$$
\mathbb{E}\left[\delta(u)^{2}\right]=\mathbb{E}\left[\int_{B(R)} u_{x}^{2} \lambda(d x)\right], \quad u \in \mathcal{P}_{0} .
$$

Covariant derivative. In addition to the gradient operator $D$, we will also need the following notion of covariant derivative operator $\widetilde{\nabla}$ defined on stochastic processes that are viewed as tangent processes on the Poisson space $\Omega$, see Privault (2001).

Definition 2.3. Let the operator $\widetilde{\nabla}$ be defined on $u \in \mathcal{P}_{0}$ as

$$
\widetilde{\nabla}_{y} u_{x}:=D_{y} u_{x}+\left\langle\mathrm{G}_{\eta}(x, y), \nabla_{x}^{\mathbb{R}^{d}} u_{x}\right\rangle_{\mathbb{R}^{d}}, \quad x, y \in B(R) .
$$

We note that from the compatibility condition (2.8) and Relation (2.9) we also have

$$
\widetilde{\nabla}_{y} u_{x}=0, \quad x \preceq y, \quad x, y \in B(R) .
$$

From the bound

$$
\begin{aligned}
& \mathbb{E}\left[\int_{B(R) \times B(R)}\left|\widetilde{\nabla}_{x} u_{y}\right| \lambda(d x) \lambda(d y)\right] \\
& \leq\|D u\|_{L^{1}(\Omega \times B(R) \times B(R))}+\mathbb{E}\left[\int_{B(R) \times B(R)}\left|\left\langle\mathrm{G}_{\eta}(x, y), \nabla_{x}^{\mathbb{R}^{d}} u_{x}\right\rangle_{\mathbb{R}^{d}}\right| \lambda(d x) \lambda(d y)\right]
\end{aligned}
$$




$$
\begin{aligned}
& \leq\|D u\|_{L^{1}(\Omega \times B(R) \times B(R))}+K_{d} \mathbb{E}\left[\int_{B(R) \times B(R)} \frac{1}{|x-y|_{\mathbb{R}^{d}}^{d-1}}\left|\nabla^{\mathbb{R}^{d}} u_{x}\right|_{\mathbb{R}^{d}} \lambda(d x) \lambda(d y)\right] \\
& \leq\|D u\|_{L^{1}(\Omega \times B(R) \times B(R))}+K_{d} v_{d} R^{\prime} \mathbb{E}\left[\int_{B(R)}\left|\nabla_{x}^{\mathbb{R}^{d}} u_{x}\right|_{\mathbb{R}^{d}} \lambda(d x)\right] \\
& =\|D u\|_{L^{1}(\Omega \times B(R) \times B(R))}+K_{d} v_{d} R^{\prime}\left\|\nabla^{\mathbb{R}^{d}} u\right\|_{L^{1}\left(\Omega \times B(R) ; \mathbb{R}^{d}\right)},
\end{aligned}
$$

we check that $\widetilde{\nabla}$ extends to the Sobolev space $\widetilde{\mathbb{D}}_{0}^{1,1}$ of predictable random fields defined as the completion of $\mathcal{P}_{0}$ under the Sobolev norm

$$
\|u\|_{\widetilde{\mathbb{D}}^{1,1}}:=\|u\|_{L^{2}\left(\Omega, W_{0}^{1,1}(B(R))\right)}+\|D u\|_{L^{1}(\Omega \times B(R) \times B(R))}, \quad u \in \mathcal{P}_{0},
$$

where $W_{0}^{1, p}(B(R))$ is the first order Sobolev space completion of $\mathcal{C}_{0}^{\infty}(B(R))$ under the norm

$$
\|f\|_{W^{1, p}(B(R))}:=\|f\|_{L^{p}(B(R))}+\left\|\nabla^{\mathbb{R}^{d}} f\right\|_{L^{p}\left(B(R) ; \mathbb{R}^{d}\right)}, \quad p \geq 1 .
$$

Commutation relation. In the sequel, we denote by $\widetilde{\mathbb{D}}_{0}^{1, \infty}$ the set of predictable random fields $u$ in $\widetilde{\mathbb{D}}_{0}^{1,1}$ that are bounded together with their covariant derivative $\widetilde{\nabla} u$.

Proposition 2.3. For $u \in \widetilde{\mathbb{D}}_{0}^{1, \infty}$ a predictable random field, we have the commutation relation

$$
D_{y} \delta(u)=u(y)+\delta\left(\widetilde{\nabla}_{y} u\right), \quad y \in B(R) .
$$

Proof. Taking $h \in \mathcal{C}_{0}^{\infty}(B(R))$, we have $\delta(h) \in \mathcal{S}$ and

$$
\begin{aligned}
D_{y} \delta(h) & =D_{y} \int_{B(R)} h(y)(\gamma(d x)-\lambda(d x)) \\
& =\int_{B(R)}\left\langle\mathrm{G}_{\eta}(x, y), \nabla_{x}^{\mathbb{R}^{d}} h(x)\right\rangle_{\mathbb{R}^{d}} \gamma(d x) \\
& =\int_{B(R)}\left\langle\mathrm{G}_{\eta}(x, y), \nabla_{x}^{\mathbb{R}^{d}} h(x)\right\rangle_{\mathbb{R}^{d}} \lambda(d x)+\delta\left(\widetilde{\nabla}_{y} h\right) \\
& =h(y)+\delta\left(\widetilde{\nabla}_{y} h\right), \quad y \in B(R),
\end{aligned}
$$

where we applied (1.9). Next, taking $u=h F \in \mathcal{P}_{0}$ a simple predictable random field, we check that $\delta(u) \in \mathcal{S}$, and by (2.5) or (6.3) we have

$$
\begin{aligned}
D_{y} \delta(F h) & =D_{y}\left(F \delta(h)-\langle h, D F\rangle_{L^{2}(B(R))}\right) \\
& =D_{y}(F \delta(h)) \\
& =\delta(h) D_{y} F+F D_{y} \delta(h) \\
& =\delta(h) D_{y} F+F\left(h(y)+\delta\left(\widetilde{\nabla}_{y} h\right)\right) \\
& =F h(y)+\delta\left(h D_{y} F+F \widetilde{\nabla}_{y} h\right) \\
& =F h(y)+\delta\left(\widetilde{\nabla}_{y}(F h)\right) \\
& =u_{y}+\delta\left(\widetilde{\nabla}_{y} u\right), \quad y \in B(R) .
\end{aligned}
$$

We conclude by the denseness of $\mathcal{P}_{0}$ in $\widetilde{\mathbb{D}}_{0}^{1,1}$ and by the closability of the operators $\widetilde{\nabla}, D$ and $\delta$. 


\section{Cumulant operators}

In the sequel, given $h$ in the standard Sobolev space $W^{1, p}(B(R))$ on $B(R)$ and $f \in L^{q}(B(R))$ with $1=p^{-1}+q^{-1}, p, q \in[1, \infty]$, we define

$$
(\widetilde{\nabla} h) f_{x}:=\int_{B(R)} f(y) \widetilde{\nabla}_{y} h(x) \lambda(d y)=\int_{B(R)} f(y)\left\langle\mathrm{G}_{\eta}(x, y), \nabla_{x}^{\mathbb{R}^{d}} h(x)\right\rangle_{\mathbb{R}^{d}} \lambda(d y),
$$

$x \in B(R)$. More generally, given $k \geq 1$ and $u \in \widetilde{\mathbb{D}}_{0}^{1,1}$ a predictable random field, we let the operator $(\widetilde{\nabla} u)^{k}$ be defined in the sense of matrix powers with continuous indices, as

$$
(\widetilde{\nabla} u)^{k} f_{y}=\int_{B(R)} \cdots \int_{B(R)}\left(\widetilde{\nabla}_{x_{k}} u_{y} \widetilde{\nabla}_{x_{k-1}} u_{x_{k}} \cdots \widetilde{\nabla}_{x_{1}} u_{x_{2}}\right) f_{x_{1}} \lambda\left(d x_{1}\right) \cdots \lambda\left(d x_{k}\right),
$$

$y \in B(R), f \in L^{2}(B(R))$.

Proposition 3.1. For any $n \in \mathbb{N}, p>1, r \in[0,1], h \in W^{1, p /(1-r)^{n-1} / r}(B(R))$ and $f \in L^{p /(1-r)^{n}}(B(R))$ we have the bound

$$
\left\|(\widetilde{\nabla} h)^{n} f\right\|_{L^{p}(B(R))} \leq\left(K_{d} v_{d} R^{\prime}\right)^{n}\|f\|_{L^{p /(1-r)^{n}}(B(R))} \prod_{j=1}^{n}\left\|\nabla^{\mathbb{R}^{d}} h\right\|_{L^{p /(1-r)^{j-1} / r}\left(B(R) ; \mathbb{R}^{d}\right)} .
$$

Proof. For $n=1$ we have

$$
\begin{aligned}
& \|(\widetilde{\nabla} h) f\|_{L^{p}(B(R))}^{p}=\int_{B(R)}\left|\int_{B(R)} f(y) \widetilde{\nabla}_{y} h(x) \lambda(d y)\right|^{p} \lambda(d x) \\
& =\int_{B(R)}\left|\int_{B(R)} f(y)\left\langle\mathrm{G}_{\eta}(x, y), \nabla_{x}^{\mathbb{R}^{d}} h(x)\right\rangle_{\mathbb{R}^{d}} \lambda(d y)\right|^{p} \lambda(d x) \\
& =\int_{B(R)}\left|\left\langle\int_{B(R)} f(y) \mathrm{G}_{\eta}(x, y) \lambda(d y), \nabla_{x}^{\mathbb{R}^{d}} h(x)\right\rangle_{\mathbb{R}^{d}}\right|^{p} \lambda(d x) \\
& \leq \int_{B(R)}\left|\int_{B(R)} f(y) \mathrm{G}_{\eta}(x, y) \lambda(d y)\right|_{\mathbb{R}^{d}}^{p}\left|\nabla_{x}^{\mathbb{R}^{d}} h(x)\right|_{\mathbb{R}^{d}}^{p} \lambda(d x) \\
& \left.=\int_{B(R)}\left|\int_{B(R)} f(y) \mathrm{G}_{\eta}(x, y) \lambda(d y)\right|_{\mathbb{R}^{d}}^{p /(1-r)} \lambda(d x)\right)^{1-r}\left(\int_{B(R)} \mid \nabla_{x}^{\left.\left.\mathbb{R}^{d} h(x)\right|_{\mathbb{R}^{d}} ^{p / r} \lambda(d x)\right)^{r}}\right. \\
& \leq\left(K_{d} v_{d} R^{\prime}\right)^{p}\|f\|_{L^{p /(1-r)}(B(R))}^{p}\left\|\nabla^{\mathbb{R}^{d}} h\right\|_{L^{p / r}\left(B(R) ; \mathbb{R}^{d}\right)}^{p},
\end{aligned}
$$

where we used the bound (1.7). Next, assuming that (3.2) holds at the rank $n \geq 1$ and using (3.3), we have

$$
\begin{aligned}
& \left\|(\widetilde{\nabla} h)^{n+1} f\right\|_{L^{p}(B(R))}=\left\|(\widetilde{\nabla} h)^{n}(\widetilde{\nabla} h) f\right\|_{L^{p}(B(R))} \\
& \quad \leq \quad\left(K_{d} v_{d} R^{\prime}\right)^{n}\|(\widetilde{\nabla} h) f\|_{L^{p /(1-r)^{n}}(B(R))} \prod_{j=1}^{n}\left\|\nabla^{\mathbb{R}^{d}} h\right\|_{L^{p /(1-r)^{j-1} / r}\left(B(R) ; \mathbb{R}^{d}\right)} \\
& \quad \leq \quad\left(K_{d} v_{d} R^{\prime}\right)^{n+1}\|f\|_{L^{p /(1-r)^{n+1}(B(R))}} \prod_{j=1}^{n+1}\left\|\nabla^{\mathbb{R}^{d}} h\right\|_{L^{p /(1-r)^{j-1} / r}\left(B(R) ; \mathbb{R}^{d}\right)},
\end{aligned}
$$


and we conclude to (3.2) by induction on $n \geq 1$.

In particular, for $r=0, f \in L^{p}(B(R)), p>1$, and $h \in W^{1,1}(B(R))$ the argument of Proposition 3.1 shows that

$$
\left\|(\widetilde{\nabla} h)^{n} f\right\|_{L^{p}(B(R))} \leq\left(K_{d} v_{d} R^{\prime}\right)^{n}\|f\|_{L^{p}(B(R))}\left\|\nabla^{\mathbb{R}^{d}} h\right\|_{L^{\infty}\left(B(R) ; \mathbb{R}^{d}\right)}^{n}, \quad n \in \mathbb{N} .
$$

We note that for $u \in \widetilde{\mathbb{D}}_{0}^{1, \infty}$ a predictable random field, the random field $(\widetilde{\nabla} u) u \in$ $\widetilde{\mathbb{D}}_{0}^{1, \infty}$ is also predictable from (2.13) and (3.1).

In the next definition we construct a family of cumulant operators which differs from the one introduced in Nourdin and Peccati (2010) on the Wiener space.

Definition 3.1. Given $k \geq 2$ and $u \in \widetilde{\mathbb{D}}_{0}^{1, \infty}$ a predictable random field we define the operators $\Gamma_{k}^{u}: \mathbb{D}_{1,1} \longrightarrow L^{1}(\Omega)$ by

$$
\Gamma_{k}^{u} F:=F\left\langle(\widetilde{\nabla} u)^{k-2} u, u\right\rangle_{L^{2}(B(R))}+\left\langle(\widetilde{\nabla} u)^{k-1} u, D F\right\rangle_{L^{2}(B(R))}, \quad F \in \mathbb{D}_{1,1} .
$$

We note that for $h$ in the space $W^{1, \infty}(B(R))$ of bounded functions in $W^{1,1}(B(R))$, and $f \in L^{p}(B(R)), p>1, m \geq 1$, we have

$$
\begin{gathered}
\left\langle h^{m},(\widetilde{\nabla} h) f\right\rangle_{L^{2}(B(R))}=\int_{B(R)} h^{m}(x) \int_{B(R)} f(y)\left\langle\mathrm{G}_{\eta}(x, y), \nabla_{x}^{\mathbb{R}^{d}} h(x)\right\rangle_{\mathbb{R}^{d}} \lambda(d y) \lambda(d x) \\
=\frac{1}{m+1} \int_{B(R)} \int_{B(R)} f(y)\left\langle\mathrm{G}_{\eta}(x, y), \nabla_{x}^{\mathbb{R}^{d}} h^{m+1}(x)\right\rangle_{\mathbb{R}^{d}} \lambda(d y) \lambda(d x) \\
=\frac{1}{m+1} \int_{B(R)} f(x) h^{m+1}(x) \lambda(d x),
\end{gathered}
$$

where we applied (1.8), hence

$$
\left\langle h^{m},(\widetilde{\nabla} h)^{n+1} f\right\rangle_{L^{2}(B(R))}=\frac{1}{m+1} \int_{B(R)} h^{m+1}(x)(\widetilde{\nabla} h)^{n} f(x) \lambda(d x),
$$

which implies by induction

$$
\left\langle(\widetilde{\nabla} h)^{n} f, h^{m}\right\rangle_{L^{2}(B(R))}=\frac{m !}{(m+n) !} \int_{B(R)} h^{m+n}(x) f(x) \lambda(d x) .
$$

In Lemma 3.2 we generalize this identity to $h$ a random field.

Lemma 3.2. For $n \in \mathbb{N}, m \geq 1, u \in \widetilde{\mathbb{D}}_{0}^{1, \infty}$ a predictable random field and $f \in$ $L^{p}(B(R)), p>1$, we have

$$
\begin{aligned}
& \left\langle(\widetilde{\nabla} u)^{n} f, u^{m}\right\rangle_{L^{2}(B(R))}=\frac{m !}{(m+n) !} \int_{B(R)} u_{x}^{m+n} f(x) \lambda(d x) \\
& +\sum_{k=1}^{n} \frac{m !}{(m+k) !}\left\langle(\widetilde{\nabla} u)^{n-k} f, D \int_{B(R)} u_{x}^{m+k} \lambda(d x)\right\rangle_{L^{2}(B(R))} .
\end{aligned}
$$

Proof. Using the adjoint $\widetilde{\nabla}^{*} u$ of $\widetilde{\nabla} u$ on $L^{2}(B(R))$ given by

$$
\left(\widetilde{\nabla}^{*} u\right) v_{y}:=\int_{B(R)}\left(\widetilde{\nabla}_{y} u_{x}\right) v_{x} \lambda(d x), \quad y \in B(R), \quad v \in L^{2}(B(R)),
$$

with the duality relation

$$
\left\langle v,\left(\widetilde{\nabla}^{*} u\right) h\right\rangle_{L^{2}(B(R))}=\langle(\widetilde{\nabla} u) v, h\rangle_{L^{2}(B(R))}, \quad h, v \in L^{2}(B(R)),
$$


we will show by induction on $k=0,1, \ldots, n$ that

$$
\begin{aligned}
& \left(\widetilde{\nabla}^{*} u\right)^{n} u_{x_{0}}^{m}=\int_{B(R)} \cdots \int_{B(R)} u_{x_{n}}^{m} \widetilde{\nabla}_{x_{0}} u_{x_{1}} \widetilde{\nabla}_{x_{1}} u_{x_{2}} \cdots \widetilde{\nabla}_{x_{n-1}} u_{x_{n}} \lambda\left(d x_{1}\right) \cdots \lambda\left(d x_{n}\right) \\
& =\sum_{i=1}^{k} \frac{m !}{(m+i) !} \\
& \quad \times \int_{B(R)} \cdots \int_{B(R)} \widetilde{\nabla}_{x_{0}} u_{x_{1}} \cdots \widetilde{\nabla}_{x_{n-i-1}} u_{x_{n-i}} D_{x_{n-i}} u_{x_{n+1}+i}^{m+i} \lambda\left(d x_{1}\right) \cdots \lambda\left(d x_{n-i-1}\right) \\
& \quad+\frac{m !}{(m+k) !} \int_{B(R)} \cdots \int_{B(R)} u_{x_{n-k}}^{m+k} \widetilde{\nabla}_{x_{0}} u_{x_{1}} \cdots \widetilde{\nabla}_{x_{n-k-1}} u_{x_{n-k}} \lambda\left(d x_{1}\right) \cdots \lambda\left(d x_{n-k}\right) .
\end{aligned}
$$

By (3.1), this relation holds for $k=0$. Next, assuming that the identity (3.5) holds for some $k \in\{0,1, \ldots, n-1\}$, and using the relation

$$
\widetilde{\nabla}_{x_{n-k-1}} u_{x_{n-k}}=D_{x_{n-k-1}} u_{x_{n-k}}+\left\langle\mathrm{G}_{\eta}\left(x_{n-k}, x_{n-k-1}\right), \widetilde{\nabla}_{x_{n-k}} u_{x_{n-k}}\right\rangle_{\mathbb{R}^{d}},
$$

$x_{n-k-1}, x_{n-k} \in B(R)$, we have

$\left(\widetilde{\nabla}^{*} u\right)^{n} u_{x_{0}}$

$$
\begin{aligned}
& =\sum_{i=1}^{k} \frac{m !}{(m+i) !} \int_{B(R)} \ldots \int_{B(R)} \widetilde{\nabla}_{x_{0}} u_{x_{1}} \cdots \widetilde{\nabla}_{x_{n-i-1}} u_{x_{n-i}} D_{x_{n-i}} u_{x_{n+1}+i}^{m+i} \lambda\left(d x_{1}\right) \cdots \lambda\left(x_{n+1-i}\right) \\
& +\frac{m !}{(m+k) !} \int_{B(R)} \ldots \int_{B(R)} u_{x_{n-k}}^{m+k} \widetilde{\nabla}_{x_{0}} u_{x_{1}} \cdots \widetilde{\nabla}_{x_{n-k-1}} u_{x_{n-k}} \lambda\left(d x_{1}\right) \cdots \lambda\left(d x_{n-k}\right) \\
& =\sum_{i=1}^{k} \frac{m !}{(m+i) !} \int_{B(R)} \ldots \int_{B(R)} \widetilde{\nabla}_{x_{0}} u_{x_{1}} \cdots \widetilde{\nabla}_{x_{n-i-1}} u_{x_{n-i}} D_{x_{n-i}} u_{x_{n+1-i}}^{m+i} \lambda\left(d x_{1}\right) \cdots \lambda\left(d x_{n+1-i}\right) \\
& +\frac{m !}{(m+k) !} \int_{B(R)} \ldots \int_{B(R)} u_{x_{n-k}}^{m+k} \widetilde{\nabla}_{x_{0}} u_{x_{1}} \cdots \widetilde{\nabla}_{x_{n-k-2}} u_{x_{n-k-1}} D_{x_{n-k-1}} u_{x_{n-k}} \lambda\left(d x_{1}\right) \cdots \lambda\left(d x_{n-k}\right) \\
& +\frac{m !}{(m+k) !} \int_{B(R)} \ldots \int_{B(R)}\left\langle G_{\eta}\left(x_{n-k}, x_{n-k-1}\right), \widetilde{\nabla}_{x_{n-k}} u_{x_{n-k}}\right\rangle_{\mathbb{R}^{d}} \\
& \times u_{x_{n-k}}^{m+k-2} \widetilde{\nabla}_{x_{0}} u_{x_{1}} \cdots \widetilde{\nabla}_{x_{n-2-k}} u_{x_{n-k-1}} \lambda\left(d x_{1}\right) \cdots \lambda\left(d x_{n-k}\right) \\
& =\sum_{i=1}^{k} \frac{m !}{(m+i) !} \int_{B(R)} \ldots \int_{B(R)} \widetilde{\nabla}_{x_{0}} u_{x_{1}} \cdots \widetilde{\nabla}_{x_{n-i-1}} u_{x_{n-i}} D_{x_{n-i}} u_{x_{n+1-i}}^{m+i} \lambda\left(d x_{1}\right) \cdots \lambda\left(d x_{n+1-i}\right) \\
& +\frac{m !}{(m+k+1) !} \int_{B(R)} \ldots \int_{B(R)} \widetilde{\nabla}_{x_{0}} u_{x_{i}} \cdots \widetilde{\nabla}_{x_{n-k}} u_{x_{n-k-1}} D_{x_{n-k-1}} u_{x_{n-k}}^{m+k+1} \lambda\left(d x_{1}\right) \cdots \lambda\left(d x_{n-k}\right) \\
& +\frac{m !}{(m+k+1) !} \int_{B(R)} \ldots \int_{B(R)} \widetilde{\nabla}_{x_{0}} u_{x_{1}} \cdots \widetilde{\nabla}_{x_{n-k-2}} u_{x_{n-k-1}} \\
& \times \int_{B(R)}\left\langle\mathrm{G}_{\eta}\left(x, x_{n-k-1}\right), \nabla_{x}^{\mathbb{R}^{d}} u_{x}^{m+k+1}\right\rangle_{\mathbb{R}^{d}} \lambda(d x) \lambda\left(d x_{1}\right) \cdots \lambda\left(d x_{n-k-1}\right) \\
& =\sum_{i=1}^{k+1} \frac{m !}{(m+i) !} \int_{B(R)} \ldots \int_{B(R)} \widetilde{\nabla}_{x_{0}} u_{x_{1}} \ldots \widetilde{\nabla}_{x_{n-i-1}} u_{x_{n-i}} D_{x_{n-i}} u_{x_{n+1-i}}^{m+i} \lambda\left(d x_{1}\right) \cdots \lambda\left(d x_{n+1-i}\right) \\
& +\frac{m !}{(m+k+1) !} \int_{B(R)} \ldots \int_{B(R)} u_{x_{n-k-1}}^{m+k+1} \widetilde{\nabla}_{x_{0}} u_{x_{1}} \cdots \widetilde{\nabla}_{x_{n-k-2}} u_{x_{n-k-1}} \lambda\left(d x_{1}\right) \cdots \lambda\left(d x_{n-k-1}\right)
\end{aligned}
$$




$$
=\sum_{i=1}^{k+1} \frac{m !}{(m+i) !}\left(\widetilde{\nabla}^{*} u\right)^{n-i} D_{x_{0}} \int_{B(R)} u_{s}^{m+i} \lambda(d s)+\frac{m !}{(m+k+1) !}\left(\widetilde{\nabla}^{*} u\right)^{n-k-1} u_{x_{0}}^{m+k+1},
$$

which shows by induction that (3.5) holds at the rank $k=n$, in particular we have

$$
\left(\widetilde{\nabla}^{*} u\right)^{n} u_{x}^{m}=\frac{m !}{(m+k) !} u_{x}^{m+n}+\sum_{i=2}^{n+1} \frac{m !}{(m+i-1) !}\left(\widetilde{\nabla}^{*} u\right)^{n+1-i} D_{x} \int_{B(R)} u_{y}^{m+i-1} \lambda(d y),
$$

$x \in B(R)$, which yields (3.4) by integration with respect to $x \in B(R)$ and duality.

As a consequence of Lemma 3.2 we have

$$
\Gamma_{k}^{u} \mathbf{1}=\int_{B(R)} \frac{u_{x}^{k}}{(k-1) !} \lambda(d x)+\sum_{i=2}^{k-1} \frac{1}{i !}\left\langle(\widetilde{\nabla} u)^{k-1-i} u, D \int_{B(R)} u_{x}^{i} \lambda(d x)\right\rangle_{L^{2}(B(R))},
$$

$k \geq 2$. Hence when $h \in W^{1, p}(B(R)), p>1$, is a deterministic function such that $\left\|\nabla^{\mathbb{R}^{d}} h\right\|_{\infty}<\infty$, we find the relation

$$
\Gamma_{k}^{h} \mathbf{1}=\frac{1}{(k-1) !} \int_{B(R)} h^{k}(x) \lambda(d x)=\frac{1}{(k-1) !} \kappa_{k}^{h}, \quad k \geq 2,
$$

which shows that $\Gamma_{k}^{h} \mathbf{1}$ coincides with the cumulant $\kappa_{k}^{h}=\int_{B(R)} h^{k}(x) \lambda(d x)$ of order $k \geq 2$ of the Poisson stochastic integral $\int_{B(R)} h(x)(\gamma(d x)-\lambda(d x))$.

\section{Edgeworth-type expansions}

Classical Edgeworth series provide expansion of the cumulative distribution function $P(F \leq x)$ of a centered random variable $F$ with $\mathbb{E}\left[F^{2}\right]=1$ around the Gaussian cumulative distribution function $\Phi(x)$, using the cumulants $\left(\kappa_{n}\right)_{n \geq 1}$ of a random variable $F$ and Hermite polynomials. Edgeworth-type expansions of the form

$$
\mathbb{E}[F g(F)]=\sum_{l=1}^{n} \frac{\kappa_{l+1}}{l !} \mathbb{E}\left[g^{(l)}(F)\right]+\mathbb{E}\left[g^{(n+1)}(F) \Gamma_{n+1} F\right], \quad n \geq 1,
$$

for $F$ a centered random variable, have been obtained by the Malliavin calculus in Nourdin and Peccati (2009a), where $\Gamma_{n+1}$ is a cumulant-type operator on the Wiener space such that $n ! \mathbb{E}\left[\Gamma_{n} F\right]$ coincides with the cumulant $\kappa_{n+1}$ of order $n+1$ of $F, n \in \mathbb{N}$, cf. Nourdin and Peccati (2010), extending the results of Barbour (1986) to the Wiener space.

In this section we establish an Edgeworth-type expansion of any finite order with an explicit remainder term for the compensated Poisson stochastic integral $\delta(u)$ of a predictable random field $\left(u_{x}\right)_{x \in B(R)}$. In the sequel we let $\langle\cdot, \cdot \cdot\rangle$ denote $\langle\cdot, \cdot\rangle_{L^{2}(B(R))}$.

Before proceeding to the statement of general expansions in Proposition 4.1, we illustrate the method with the derivation of an expansion of order one for a deterministic integrand $f$. By the duality relation (2.6) between $D$ and $\delta$, the chain rule of derivation for $D$ and the commutation relation (2.14) we get, for $g \in \mathcal{C}_{b}^{2}(\mathbb{R})$ and $f \in W_{0}^{1,1}(B(R))$ such that $\left\|\nabla^{\mathbb{R}^{d}} f\right\|_{\infty}<\infty$,

$$
\begin{aligned}
& \mathbb{E}[\delta(f) g(\delta(f))]=\mathbb{E}\left[\langle f, D \delta(f)\rangle g^{\prime}(\delta(f))\right] \\
& \quad=\mathbb{E}\left[\langle f, f\rangle g^{\prime}(\delta(f))\right]+\mathbb{E}\left[\left\langle f, \delta\left(\widetilde{\nabla}^{*} f\right)\right\rangle g^{\prime}(\delta(f))\right]
\end{aligned}
$$




$$
\begin{aligned}
= & \mathbb{E}\left[\langle f, f\rangle g^{\prime}(\delta(f))\right]+\mathbb{E}\left[\left\langle\widetilde{\nabla}^{*} f, D\left(g^{\prime}(\delta(f)) f\right)\right\rangle\right] \\
= & \mathbb{E}\left[\langle f, f\rangle g^{\prime}(\delta(f))\right]+\mathbb{E}\left[\langle(\widetilde{\nabla} f) f, D \delta(f)\rangle g^{\prime \prime}(\delta(f))\right] \\
= & \mathbb{E}\left[\langle f, f\rangle g^{\prime}(\delta(f))\right]+\frac{1}{2} \int_{B(R)} f^{3}(x) \lambda(d x) \mathbb{E}\left[g^{\prime \prime}(\delta(f))\right] \\
& +\mathbb{E}\left[\left\langle(\widetilde{\nabla} f) f, \delta\left(\widetilde{\nabla}^{*} f\right)\right\rangle g^{\prime \prime}(\delta(f))\right] \\
= & \kappa_{2}^{f} \mathbb{E}\left[g^{\prime}(\delta(f))\right]+\frac{1}{2} \kappa_{3}^{f} \mathbb{E}\left[g^{\prime \prime}(\delta(f))\right]+\mathbb{E}\left[g^{\prime \prime}(\delta(f)) \delta\left((\widetilde{\nabla} f)^{2} f\right)\right],
\end{aligned}
$$

since by Lemma 3.2 we have

$$
\langle(\widetilde{\nabla} f) f, f\rangle=\frac{1}{2} \int_{B(R)} f^{3}(x) \lambda(d x)=\frac{1}{2} \kappa_{3}^{f} .
$$

In the next proposition we derive general Edgeworth-type expansions for predictable integrand processes $\left(u_{x}\right)_{x \in \mathbb{R}^{d}}$.

Proposition 4.1. Let $u \in \widetilde{\mathbb{D}}_{0}^{1, \infty}$ and $n \geq 0$. For all $g \in \mathcal{C}_{b}^{n+1}(\mathbb{R})$ and bounded $G \in \mathbb{D}_{1,1}$ we have

$$
\begin{aligned}
& \mathbb{E}[G \delta(u) g(\delta(u))]=\mathbb{E}[\langle u, D G\rangle g(\delta(u))]+\sum_{k=1}^{n} \mathbb{E}\left[g^{(k)}(\delta(u)) \Gamma_{k+1}^{u} G\right] \\
& +\mathbb{E}\left[G g^{(n+1)}(\delta(u))\left(\int_{B(R)} \frac{u_{x}^{n+2}}{(n+1) !} \lambda(d x)+\sum_{k=2}^{n+1}\left\langle(\widetilde{\nabla} u)^{n+1-k} u, D \int_{B(R)} \frac{u_{x}^{k}}{k !} \lambda(d x)\right\rangle\right)\right] \\
& +\mathbb{E}\left[G g^{(n+1)}(\delta(u))\left\langle(\widetilde{\nabla} u)^{n} u, \delta\left(\widetilde{\nabla}^{*} u\right)\right\rangle\right] .
\end{aligned}
$$

Proof. By the duality relation (2.6) between $D$ and $\delta$, the chain rule of derivation for $D$ and the commutation relation (2.14), we get

$$
\begin{aligned}
\mathbb{E} & {\left[G\left\langle(\widetilde{\nabla} u)^{k} u, D \delta(u)\right\rangle g(\delta(u))\right]-\mathbb{E}\left[G\left\langle(\widetilde{\nabla} u)^{k+1} u, D \delta(u)\right\rangle g^{\prime}(\delta(u))\right] } \\
= & \mathbb{E}\left[G\left\langle(\widetilde{\nabla} u)^{k} u, u\right\rangle g(\delta(u))\right]+\mathbb{E}\left[G\left\langle(\widetilde{\nabla} u)^{k} u, \delta\left(\widetilde{\nabla}^{*} u\right)\right\rangle g(\delta(u))\right] \\
& -\mathbb{E}\left[G\left\langle(\widetilde{\nabla} u)^{k+1} u, D \delta(u)\right\rangle g^{\prime}(\delta(u))\right] \\
= & \mathbb{E}\left[G\left\langle(\widetilde{\nabla} u)^{k} u, u\right\rangle g(\delta(u))\right]+\mathbb{E}\left[\left\langle\widetilde{\nabla}^{*} u, D\left(G g(\delta(u))(\widetilde{\nabla} u)^{k} u\right)\right]\right\rangle \\
& -\mathbb{E}\left[G\left\langle(\widetilde{\nabla} u)^{k+1} u, D \delta(u)\right\rangle g^{\prime}(\delta(u))\right] \\
= & \mathbb{E}\left[G\left\langle(\widetilde{\nabla} u)^{k} u, u\right\rangle g(\delta(u))\right]+\mathbb{E}\left[\left\langle(\widetilde{\nabla} u)^{k+1} u, D G\right\rangle g(\delta(u))\right] \\
& +\mathbb{E}\left[G\left\langle\widetilde{\nabla}^{*} u, D\left((\widetilde{\nabla} u)^{k} u\right)\right\rangle g(\delta(u))\right] \\
= & \mathbb{E}\left[g(\delta(u)) \Gamma_{k+2}^{u} G\right],
\end{aligned}
$$

where we used (2.9) and (2.13). Therefore, we have

$$
\begin{aligned}
& \mathbb{E}[G \delta(u) g(\delta(u))]=\mathbb{E}[\langle u, D(G g(\delta(u)))\rangle] \\
& =\mathbb{E}\left[G\langle u, D \delta(u)\rangle g^{\prime}(\delta(u))\right]+\mathbb{E}[\langle u, D G\rangle g(\delta(u))] \\
& =\mathbb{E}[\langle u, D G\rangle g(\delta(u))]+\mathbb{E}\left[G g^{(n+1)}(\delta(u))\left\langle(\widetilde{\nabla} u)^{n} u, D \delta(u)\right\rangle\right] \\
& +\sum_{k=0}^{n-1}\left(\mathbb{E}\left[G g^{(k+1)}(\delta(u))\left\langle(\widetilde{\nabla} u)^{k} u, D \delta(u)\right\rangle\right]-\mathbb{E}\left[G g^{(k+2)}(\delta(u))\left\langle(\widetilde{\nabla} u)^{k+1} u, D \delta(u)\right\rangle\right]\right)
\end{aligned}
$$




$$
\begin{aligned}
= & \mathbb{E}[\langle u, D G\rangle g(\delta(u))]+\sum_{k=1}^{n} \mathbb{E}\left[g^{(k)}(\delta(u)) \Gamma_{k+1}^{u} G\right] \\
& +\mathbb{E}\left[G g^{(n+1)}(\delta(u))\left\langle(\widetilde{\nabla} u)^{n} u, D \delta(u)\right\rangle\right] \\
= & \mathbb{E}[\langle u, D G\rangle g(\delta(u))]+\sum_{k=1}^{n} \mathbb{E}\left[g^{(k)}(\delta(u)) \Gamma_{k+1}^{u} G\right] \\
& +\mathbb{E}\left[G g^{(n+1)}(\delta(u))\left\langle(\widetilde{\nabla} u)^{n} u, u\right\rangle\right]+\mathbb{E}\left[G g^{(n+1)}(\delta(u))\left\langle(\widetilde{\nabla} u)^{n} u, \delta\left(\widetilde{\nabla}^{*} u\right)\right\rangle\right],
\end{aligned}
$$

and we conclude by Lemma 3.2.

When $f \in W_{0}^{1,1}(B(R))$ is a deterministic function such that $\left\|\nabla^{d} f\right\|_{\infty}<\infty$, and $g \in \mathcal{C}_{b}^{\infty}(\mathbb{R})$, Proposition 4.1 shows that

$$
\begin{aligned}
\mathbb{E} & {[\delta(f) g(\delta(f))] } \\
& =\sum_{k=1}^{n+1} \frac{1}{k !} \int_{B(R)} f^{k+1}(x) \lambda(d x) \mathbb{E}\left[g^{(k)}(\delta(f))\right]+\mathbb{E}\left[g^{(n+1)}(\delta(f))\left\langle(\widetilde{\nabla} f)^{n} f, \delta\left(\widetilde{\nabla}^{*} f\right)\right\rangle\right] \\
& =\sum_{k=1}^{n+1} \frac{1}{k !} \kappa_{k+1}^{f} \mathbb{E}\left[g^{(k)}(\delta(f))\right]+\mathbb{E}\left[g^{(n+1)}(\delta(f)) \delta\left((\widetilde{\nabla} f)^{n+1} f\right)\right], \quad n \geq 0,
\end{aligned}
$$

with, by Proposition 3.1 applied with $p=2$ and $r=0$,

$$
\begin{aligned}
\mathbb{E}\left[\left|\delta\left((\widetilde{\nabla} f)^{n+1} f\right)\right|\right] & \leq \sqrt{\mathbb{E}\left[\left|\delta\left((\widetilde{\nabla} f)^{n+1} f\right)\right|^{2}\right]} \\
& =\left\|(\widetilde{\nabla} f)^{n+1} f\right\|_{L^{2}(B(R))} \\
& \leq\left(K_{d} v_{d} R^{\prime}\right)^{n+1}\|f\|_{L^{2}(B(R))}\|\widetilde{\nabla} f\|_{L^{\infty}\left(B(R) ; \mathbb{R}^{d}\right)}^{n+1} .
\end{aligned}
$$

In addition, as $n$ tends to $+\infty$ we have

$$
\begin{aligned}
\mathbb{E}[\delta(f) g(\delta(f))] & =\sum_{k=1}^{\infty} \frac{1}{k !} \int_{B(R)} f^{k+1}(x) \lambda(d x) \mathbb{E}\left[g^{(k)}(\delta(f))\right] \\
& =\sum_{k=1}^{\infty} \frac{1}{k !} \int_{B(R)} f^{k+1}(x) \lambda(d x) \mathbb{E}\left[g^{(k)}(\delta(f))\right] \\
& =\mathbb{E}\left[\int_{B(R)} f(x)(g(\delta(f)+f(x))-g(\delta(f))) \lambda(d x)\right]
\end{aligned}
$$

provided that the derivatives of $g$ decay fast enough, which is a particular instance of the standard integration by parts identity for finite difference operators on the Poisson space, see e.g. Lemma 2.9 in Peccati et al. (2010) or Lemma 5 in Bourguin and Peccati (2016).

\section{Stein approximation}

Applying Proposition 4.1 with $n=0$ and $G=1$ to the solution $g_{x}$ of the Stein equation

$$
\mathbf{1}_{(-\infty, x]}(z)-\Phi(z)=g_{x}^{\prime}(z)-z g_{x}(z), \quad z \in \mathbb{R}
$$

and letting $u \in \widetilde{\mathbb{D}}_{0}^{1,1}$ be a predictable random field, this gives the expansion

$$
P(\delta(u) \leq x)-\Phi(x)=\mathbb{E}\left[g_{x}^{\prime}(\delta(u))\langle u, u\rangle-\delta(u) g_{x}(\delta(u))\right]
$$




$$
=\mathbb{E}\left[(1-\langle u, u\rangle) g_{x}^{\prime}(\delta(u))\right]+\mathbb{E}\left[\langle u, \delta(\widetilde{\nabla} u)\rangle g_{x}^{\prime}(\delta(u))\right],
$$

around the Gaussian cumulative distribution function $\Phi(x)$, with $\left\|g_{x}\right\|_{\infty} \leq \sqrt{2 \pi} / 4$ and $\left\|g_{x}^{\prime}\right\|_{\infty} \leq 1, x \in \mathbb{R}$, by Lemma 2.2-(v) of Chen et al. (2011). The next result follows from the application of Proposition 4.1 with $n=1$ and $G=1$.

Proposition 5.1. For any random field $u \in \widetilde{\mathbb{D}}_{0}^{1, \infty}$ we have $d_{W}(\delta(u), \mathcal{N})$

$$
\begin{aligned}
\leq & \mathbb{E}\left[\left|1-\langle u, u\rangle-\left\langle\widetilde{\nabla}^{*} u, D u\right\rangle\right|\right]+\mathbb{E}\left[\left|\int_{B(R)} u_{x}^{3} \lambda(d x)+\left\langle u, D \int_{B(R)} u_{x}^{2} \lambda(d x)\right\rangle\right|\right] \\
& +2 \mathbb{E}\left[\left|\left\langle(\widetilde{\nabla} u) u, \delta\left(\widetilde{\nabla}^{*} u\right)\right\rangle\right|\right] .
\end{aligned}
$$

Proof. For $n=1$ and $G=1$, Proposition 4.1 shows that

$$
\begin{aligned}
\mathbb{E}[\delta(u) g(\delta(u))]= & \mathbb{E}\left[g^{\prime}(\delta(u))\left(\langle u, u\rangle+\left\langle\widetilde{\nabla}^{*} u, D u\right\rangle\right)\right] \\
& +\frac{1}{2} \mathbb{E}\left[g^{\prime \prime}(\delta(u))\left(\int_{B(R)} u_{x}^{3} \lambda(d x)+\left\langle u, D \int_{B(R)} u_{x}^{2} \lambda(d x)\right\rangle\right)\right] \\
& +\mathbb{E}\left[g^{\prime \prime}(\delta(u))\langle(\widetilde{\nabla} u) u, \delta(\widetilde{\nabla} u)\rangle\right] .
\end{aligned}
$$

Let $h: \mathbb{R} \rightarrow[0,1]$ be a continuous function with bounded derivative. Using the solution $g_{h} \in \mathcal{C}_{b}^{1}(\mathbb{R})$ of the Stein equation

$$
h(z)-\mathbb{E}[h(\mathcal{N})]=g^{\prime}(z)-z g(z), \quad z \in \mathbb{R},
$$

with the bounds $\left\|g_{h}^{\prime}\right\|_{\infty} \leq\left\|h^{\prime}\right\|_{\infty}$ and $\left\|g_{h}^{\prime \prime}\right\|_{\infty} \leq 2\left\|h^{\prime}\right\|_{\infty}, x \in \mathbb{R}$, cf. Lemma 1.2-(v) of Nourdin and Peccati (2009b) and references therein, we have

$$
\begin{aligned}
\mathbb{E}[h(\delta(u))]- & \mathbb{E}[h(\mathcal{N})]=\mathbb{E}\left[\delta(u) g_{h}(\delta(u))-g_{h}^{\prime}(\delta(u))\right] \\
= & \mathbb{E}\left[g_{h}^{\prime}(\delta(u))\left(\langle u, u\rangle+\left\langle\widetilde{\nabla}^{*} u, D u\right\rangle-1\right)\right] \\
& +\frac{1}{2} \mathbb{E}\left[g^{\prime \prime}(\delta(u))\left(\int_{B(R)} u_{x}^{3} \lambda(d x)+\left\langle u, D \int_{B(R)} u_{x}^{2} \lambda(d x)\right\rangle\right)\right] \\
& +2 \mathbb{E}\left[g_{h}^{\prime \prime}(\delta(u))\left\langle(\widetilde{\nabla} u) u, \delta\left(\widetilde{\nabla}^{*} u\right)\right\rangle\right],
\end{aligned}
$$

hence

$$
\begin{gathered}
|\mathbb{E}[\delta(u) h(\delta(u))]-\mathbb{E}[h(\mathcal{N})]| \leq\left\|h^{\prime}\right\|_{\infty} \mathbb{E}\left[\left|1-\langle u, u\rangle-\left\langle\widetilde{\nabla}^{*} u, D u\right\rangle\right|\right] \\
+\left\|h^{\prime}\right\|_{\infty} \mathbb{E}\left[\left|\int_{B(R)} u_{x}^{3} \lambda(d x)+\left\langle u, D \int_{B(R)} u_{x}^{2} \lambda(d x)\right\rangle\right|\right] \\
+2\left\|h^{\prime}\right\|_{\infty} \mathbb{E}\left[\left|\left\langle(\widetilde{\nabla} u) u, \delta\left(\widetilde{\nabla}^{*} u\right)\right\rangle\right|\right],
\end{gathered}
$$

which yields (5.2).

As a consequence of Proposition 5.1 and the Itô isometry (2.12) we have the following corollary.

Corollary 5.1. For $u \in \widetilde{\mathbb{D}}_{0}^{1, \infty}$ we have

$$
\begin{aligned}
d_{W}(\delta(u), \mathcal{N}) \leq & |1-\operatorname{Var}[\delta(u)]|+\sqrt{\operatorname{Var}\left[\|u\|_{L^{2}(B(R))}^{2}\right]} \\
& +\mathbb{E}\left[\left|\int_{B(R)} u_{x}^{3} \lambda(d x)+\left\langle u, D \int_{B(R)} u_{x}^{2} \lambda(d x)\right\rangle\right|\right]
\end{aligned}
$$




$$
+\mathbb{E}\left[\left|\left\langle\widetilde{\nabla}^{*} u, D u\right\rangle\right|\right]+2 \mathbb{E}\left[\left|\left\langle(\widetilde{\nabla} u) u, \delta\left(\widetilde{\nabla}^{*} u\right)\right\rangle\right|\right] .
$$

Proof. By the Itô isometry (2.12) we have

$$
\operatorname{Var}[\delta(u)]=\mathbb{E}\left[\left(\int_{B(R)} u_{x}(\gamma(d x)-\lambda(d x))\right)^{2}\right]=\mathbb{E}[\langle u, u\rangle]
$$

hence

$$
\begin{aligned}
\mathbb{E} & {\left[\left|1-\langle u, u\rangle-\left\langle\widetilde{\nabla}^{*} u, D u\right\rangle\right|\right] } \\
& \leq \mathbb{E}[|1-\mathbb{E}[\langle u, u\rangle]|]+\mathbb{E}[|\langle u, u\rangle-\mathbb{E}[\langle u, u\rangle]|]+\mathbb{E}\left[\left|\left\langle\widetilde{\nabla}^{*} u, D u\right\rangle\right|\right] \\
& =|1-\operatorname{Var}[\delta(u)]|+\sqrt{\mathbb{E}\left[(\langle u, u\rangle-\mathbb{E}[\langle u, u\rangle])^{2}\right]}+\mathbb{E}\left[\left|\left\langle\widetilde{\nabla}^{*} u, D u\right\rangle\right|\right] \\
& =|1-\operatorname{Var}[\delta(u)]|+\sqrt{\operatorname{Var}\left[\|u\|_{L^{2}(B(R))}^{2}\right]}+\mathbb{E}\left[\left|\left\langle\widetilde{\nabla}^{*} u, D u\right\rangle\right|\right] .
\end{aligned}
$$

In particular, when $\operatorname{Var}[\delta(u)]=1$, Corollary 5.1 shows that

$$
\begin{aligned}
d_{W}(\delta(u), \mathcal{N}) \leq & \sqrt{\operatorname{Var}\left[\|u\|_{L^{2}(B(R))}^{2}\right]} \\
& +\mathbb{E}\left[\left|\int_{B(R)} u_{x}^{3} \lambda(d x)+\left\langle u, D \int_{B(R)} u_{x}^{2} \lambda(d x)\right\rangle\right|\right] \\
& +\mathbb{E}\left[\left|\left\langle\widetilde{\nabla}^{*} u, D u\right\rangle\right|\right]+2 \mathbb{E}\left[\left|\left\langle(\widetilde{\nabla} u) u, \delta\left(\widetilde{\nabla}^{*} u\right)\right\rangle\right|\right] .
\end{aligned}
$$

When $f \in W_{0}^{1, \infty}(B(R))$ is a deterministic function we have

$$
\operatorname{Var}[\delta(f)]=\mathbb{E}\left[\left(\int_{B(R)} f(x)(\gamma(d x)-\lambda(d x))\right)^{2}\right]=\int_{B(R)} f^{2}(x) \lambda(d x),
$$

and Corollary 5.1 shows that

$$
d_{W}(\delta(f), \mathcal{N}) \leq\left|1-\int_{B(R)} f^{2}(x) \lambda(d x)\right|+\left|\int_{B(R)} f^{3}(x) \lambda(d x)\right|+2 \mathbb{E}\left[\left|\delta\left((\widetilde{\nabla} f)^{2} f\right)\right|\right] .
$$

Given the bound

$$
\begin{aligned}
\mathbb{E}\left[\left|\delta\left((\widetilde{\nabla} f)^{2} f\right)\right|\right] & \leq \sqrt{\mathbb{E}\left[\left|\delta\left((\widetilde{\nabla} f)^{2} f\right)\right|^{2}\right]} \\
& =\left\|(\widetilde{\nabla} f)^{2} f\right\|_{L^{2}(B(R))} \\
& \leq\left(K_{d} v_{d} R^{\prime}\right)^{2}\|f\|_{L^{2}(B(R))}\left\|\nabla^{\mathbb{R}^{d}} f\right\|_{L^{\infty}\left(B(R) ; \mathbb{R}^{d}\right)}^{2}
\end{aligned}
$$

obtained from Proposition 3.1 with $p=2$ and $r=0, f \in W_{0}^{1, \infty}(B(R))$, we also have the following corollary.

Corollary 5.2. For $f \in W_{0}^{1, \infty}(B(R))$ we have

$$
\begin{gathered}
d_{W}\left(\int_{B(R)} f(x)(\gamma(d x)-\lambda(d x)), \mathcal{N}\right) \leq\left|1-\|f\|_{L^{2}(B(R))}^{2}\right|+\left|\int_{B(R)} f^{3}(x) \lambda(d x)\right| \\
+2\left(K_{d} v_{d} R^{\prime}\right)^{2}\|f\|_{L^{2}(B(R))}\left\|\nabla^{\mathbb{R}^{d}} f\right\|_{L^{\infty}\left(B(R) ; \mathbb{R}^{d}\right)}^{2} .
\end{gathered}
$$


In particular, if $\|f\|_{L^{2}(B(R))}=1$ we find

$$
\begin{aligned}
d_{W}\left(\int_{B(R)} f(x)(\gamma(d x)-\lambda(d x)), \mathcal{N}\right) \leq & \left|\int_{B(R)} f^{3}(x) \lambda(d x)\right| \\
& +2\left(K_{d} v_{d} R^{\prime}\right)^{2}\left\|\nabla^{\mathbb{R}^{d}} f\right\|_{L^{\infty}\left(B(R) ; \mathbb{R}^{d}\right)}^{2}
\end{aligned}
$$

As an example, consider $f_{k}$ given on $B\left(k^{1 / d} R\right)$ by

$$
f_{k}(x):=\frac{1}{C \sqrt{k}} g\left(\frac{|x|_{\mathbb{R}^{d}}}{k^{1 / d}}\right), \quad x \in B\left(k^{1 / d} R\right),
$$

where $g \in \mathcal{C}^{1}([0, R])$ is such that $g(R)=0$, and

$$
C^{2}:=v_{d} \int_{0}^{R} g^{2}(r) r^{d-1} d r,
$$

so that $f_{k} \in L^{2}\left(B\left(k^{1 / d} R\right)\right)$ with

$$
\|f\|_{L^{2}\left(B\left(k^{1 / d} R\right)\right)}^{2}=\frac{v_{d}}{C^{2} k} \int_{0}^{k^{1 / d} R} g^{2}\left(\frac{r}{k^{1 / d}}\right) r^{d-1} d r=\frac{v_{d}}{C^{2}} \int_{0}^{R} g^{2}(r) r^{d-1} d r=1,
$$

and

$$
\int_{B\left(k^{1 / d} R\right)} f_{k}^{3}(x) d x=\frac{1}{C^{3} k^{3 / 2}} \int_{0}^{k^{1 / d} R} g^{3}\left(r k^{-1 / d}\right) r^{d-1} d r=\frac{1}{C^{3} \sqrt{k}} \int_{0}^{R} g^{3}(r) r^{d-1} d r,
$$

$k \geq 1$. We have

$$
\left\|\nabla^{\mathbb{R}^{d}} f_{k}\right\|_{L^{\infty}\left(B(R) ; \mathbb{R}^{d}\right)}^{2} \leq \frac{\left\|g^{\prime}\right\|_{\infty}^{2} d}{C^{2} k^{1+2 / d}}
$$

hence

$$
\begin{gathered}
d_{W}\left(\int_{B(R)} f_{k}(x)(\gamma(d x)-\lambda(d x)), \mathcal{N}\right) \\
\leq\left|\int_{B(R)} f_{k}^{3}(x) \lambda(d x)\right|+\frac{2\left(K_{d} v_{d} k^{1 / d} R^{\prime}\right)^{2} d}{k^{1+2 / d} C^{2}}\left\|g^{\prime}\right\|_{\infty}^{2} \\
\leq \frac{v_{d}}{C^{3} \sqrt{k}}\left|\int_{0}^{R} g^{3}(r) r^{d-1} d r\right|+\frac{2\left(K_{d} v_{d} R^{\prime}\right)^{2} d}{k C^{2}}\left\|g^{\prime}\right\|_{\infty}^{2} .
\end{gathered}
$$

In particular, if $g$ satisfies the condition

$$
\int_{0}^{R} g^{3}(r) r^{d-1} d r=0,
$$

then we find the $O(1 / k)$ convergence rate

$$
d_{W}\left(\int_{B(R)} f_{k}(x)(\gamma(d x)-\lambda(d x)), \mathcal{N}\right) \leq \frac{2\left(K_{d} v_{d} R^{\prime}\right)^{2} d}{k C^{2}}\left\|g^{\prime}\right\|_{\infty}^{2}, \quad k \geq 1 .
$$

For example, taking

$$
f_{k}(x):=\frac{1}{C \sqrt{k}} g\left(\frac{|x|_{\mathbb{R}^{d}}}{k^{1 / d}}\right)=\frac{1}{C \sqrt{k}}\left(h_{1}\left(\frac{|x|_{\mathbb{R}^{d}}}{k^{1 / d}}\right)-a h_{2}\left(\frac{|x|_{\mathbb{R}^{d}}}{k^{1 / d}}\right)\right), \quad x \in B\left(k^{1 / d} R\right),
$$


with $a \in \mathbb{R}, h_{1}, h_{2} \in \mathcal{C}^{1}([0, R])$ such that $h_{1}(R)=h_{2}(R)=0$, and

$$
C^{2}:=\int_{0}^{R}\left(h_{1}(r)-a h_{2}(r)\right)^{2} r^{d-1} d r>0
$$

we can choose $a \in \mathbb{R}$ satisfying the cubic equation

$$
\begin{aligned}
\int_{B(R)} g^{3}(r) r^{d-1} d r= & a^{3} \int_{0}^{R} h_{2}^{3}(r) r^{d-1} d r+3 a^{2} \int_{0}^{R} h_{1}(r) h_{2}^{2}(r) r^{d-1} d r \\
& -3 a \int_{0}^{R} h_{1}^{2}(r) h_{2}(r) r^{d-1} d r+\int_{0}^{R} h_{1}^{3}(r) r^{d-1} d r \\
= & 0,
\end{aligned}
$$

which yields the bound

$$
d_{W}\left(\int_{B\left(k^{1 / d} R\right)} f_{k}(x)(\gamma(d x)-\lambda(d x)), \mathcal{N}\right) \leq \frac{c\left(a, d, h_{1}, h_{2}\right)}{k}, \quad k \geq 1,
$$

from (1.5), where $c\left(a, d, h_{1}, h_{2}\right)$ depends only on $a \in \mathbb{R}, d \geq 2$ and $h_{1}, h_{2} \in$ $\mathcal{C}^{1}([0, R])$, whereas $(1.3)$ can only yield the standard Berry-Esseen convergence rate (1.4) as $\int_{0}^{R}|g(r)|^{3} r^{d-1} d r>0$.

\section{Appendix}

Proof of Proposition 2.1.

As a consequence of (1.8) and (2.2) we have

$$
\begin{aligned}
f_{n} & \left(x_{1}, \ldots, x_{i-1}, y, x_{i+1}, \ldots x_{n}\right)-f_{n-1}\left(x_{1}, \ldots, x_{i-1}, x_{i+1}, \ldots, x_{n}\right) \\
= & f_{n}\left(x_{1}, \ldots, x_{i-1}, y, x_{i+1}, \ldots x_{n}\right) \\
& -f_{n-1}\left(x_{1}, \ldots, x_{i-1}, x_{i+1}, \ldots, x_{n}\right) \int_{B\left(R^{\prime}\right) \backslash B(R)} \eta(x) \lambda(d x) \\
= & f_{n}\left(x_{1}, \ldots, x_{i-1}, y, x_{i+1}, \ldots x_{n}\right) \\
& -\int_{B\left(R^{\prime}\right) \backslash B(R)} \eta(x) f_{n}\left(x_{1}, \ldots, x_{i-1}, x, x_{i+1}, \ldots, x_{n}\right) \lambda(d x) \\
= & \int_{B\left(R^{\prime}\right)}\left\langle G\left(x_{i}, y\right), \nabla_{x_{i}}^{\mathbb{R}^{d}} f_{n}\left(x_{1}, \ldots, x_{n}\right)\right\rangle_{\mathbb{R}^{d}} \lambda\left(d x_{i}\right) \\
= & \int_{B(R)}\left\langle G\left(x_{i}, y\right), \nabla_{x_{i}}^{\mathbb{R}^{d}} f_{n}\left(x_{1}, \ldots, x_{n}\right)\right\rangle_{\mathbb{R}^{d}} \lambda\left(d x_{i}\right)
\end{aligned}
$$

$x_{1}, \ldots, x_{i-1}, y, x_{i+1}, \ldots, x_{n} \in B\left(R^{\prime}\right)$. Recall that for all $F \in \mathcal{S}$ of the form (2.1) we have

$$
\mathbb{E}[F]=e^{-\lambda(B(R))} \sum_{n=0}^{\infty} \frac{1}{n !} \int_{B(R)} \cdots \int_{B(R)} f_{n}\left(x_{1}, \ldots, x_{n}\right) \lambda\left(d x_{1}\right) \cdots \lambda\left(d x_{n}\right) .
$$

Hence, using (6.1), for $g \in \mathcal{C}_{0}^{1}(B(R))$ and $F$ of the form (2.1) we have

$$
\mathbb{E}\left[\int_{B(R)} g(y) D_{y} F \lambda(d y)\right]
$$




$$
\begin{aligned}
& =\mathbb{E}\left[\sum_{n=1}^{\infty} \mathbf{1}_{\{\gamma(B(R))=n\}} \sum_{i=1}^{n} \int_{B(R)} g(y)\left\langle\mathrm{G}_{\eta}\left(X_{i}, y\right), \nabla_{X_{i}}^{\mathbb{R}^{d}} f\left(X_{1}, \ldots, X_{n}\right)\right\rangle_{\mathbb{R}^{d}} \lambda(d y)\right] \\
& =e^{-\lambda(B(R))} \sum_{n=1}^{\infty} \frac{1}{n !} \int_{B(R)} \cdots \\
& \cdots \int_{B(R)} \sum_{i=1}^{n} \int_{B(R)} g(y)\left\langle\mathrm{G}_{\eta}\left(x_{i}, y\right), \nabla_{x_{i}}^{\mathbb{R}^{d}} f_{n}\left(x_{1}, \ldots, x_{n}\right)\right\rangle_{\mathbb{R}^{d}} \lambda(d y) \lambda\left(d x_{1}\right) \cdots \lambda\left(d x_{n}\right) \\
& =e^{-\lambda(B(R))} \sum_{n=1}^{\infty} \frac{1}{n !} \int_{B(R)} \ldots \\
& \cdots \int_{B(R)} \sum_{i=1}^{n} \int_{B(R)} g(y) f_{n}\left(x_{1}, \ldots, x_{i-1}, y, x_{i+1}, \ldots, x_{n}\right) \lambda\left(d x_{1}\right) \cdots \lambda(d y) \cdots \lambda\left(d x_{n}\right) \\
& -e^{-\lambda(B(R))} \sum_{n=1}^{\infty} \frac{1}{n !} \int_{B(R)} \ldots \\
& \cdots \int_{B(R)} \sum_{i=1}^{n} \int_{B(R)} g(y) \lambda(d y) f_{n-1}\left(x_{1}, \ldots, x_{n-1}\right) \lambda\left(d x_{1}\right) \cdots \lambda\left(d x_{n-1}\right) \\
& =e^{-\lambda(B(R))} \sum_{n=1}^{\infty} \frac{1}{n !} \int_{B(R)} \ldots \\
& \cdots \int_{B(R)}\left(\sum_{i=1}^{n} g\left(x_{i}\right)-\int_{B(R)} g(y) \lambda(d y)\right) f_{n}\left(x_{1}, \ldots, x_{n}\right) \lambda\left(d x_{1}\right) \cdots \lambda\left(d x_{n}\right) \\
& =\mathbb{E}\left[F\left(\int_{B(R)} g(x)(\gamma(d x)-\lambda(d x))\right)\right] \text {. }
\end{aligned}
$$

Next, for $u$ of the form (2.4), we check by a standard argument that

$$
\begin{aligned}
\mathbb{E}[\langle u, D F\rangle] & =\sum_{i=1}^{n} \mathbb{E}\left[G_{i}\left\langle g_{i}, D F\right\rangle\right] \\
& =\sum_{i=1}^{n}\left(\mathbb{E}\left[\left\langle g_{i}, D\left(F G_{i}\right)\right\rangle-F\left\langle g_{i}, D G_{i}\right\rangle\right]\right) \\
& =\mathbb{E}\left[F \sum_{i=1}^{n}\left(G_{i} \int_{B(R)} g_{i}(x)(\gamma(d x)-\lambda(d x))-\left\langle g_{i}, D G_{i}\right\rangle\right)\right] \\
& =\mathbb{E}[F \delta(u)] .
\end{aligned}
$$

Proof of Proposition 2.2. Taking $u \in \mathcal{P}_{0}$ a predictable random field of the form (2.7) we note that by (2.3) and the compatibility condition (2.10) we have

$$
g_{i}(y) D_{y} F_{i}=0, \quad y \in B(R), \quad i=1, \ldots, n,
$$


hence by (2.5) we have

$$
\begin{aligned}
\delta(u) & =\delta\left(\sum_{i=1}^{n} F_{i} g_{i}\right)=\sum_{i=1}^{n} F_{i} \delta\left(g_{i}\right) \\
& =\sum_{i=1}^{n} F_{i} \int_{B(R)} g_{i}(x)(\gamma(d x)-\lambda(d x)) \\
& =\int_{B(R)} u_{x}(\gamma(d x)-\lambda(d x)),
\end{aligned}
$$

showing that $\delta(u)$ coincides with the Poisson stochastic integral of $\left(u_{x}\right)_{x \in B(R)}$. Regarding the isometry relation (2.12), we have

$$
\begin{aligned}
\mathbb{E}\left[\delta(u)^{2}\right] & =\mathbb{E}\left[\left(\sum_{i=1}^{n} F_{i} \int_{B(R)} g_{i}(x)(\gamma(d x)-\lambda(d x))\right)^{2}\right] \\
= & \mathbb{E}\left[\sum_{i, j=1}^{n} F_{i} F_{j} \int_{B(R)} g_{i}(x)(\gamma(d x)-\lambda(d x)) \int_{B(R)} g_{j}(x)(\gamma(d x)-\lambda(d x))\right] \\
= & 2 \mathbb{E}\left[\sum_{1 \leq i<j \leq n} F_{i} \int_{B(R)} g_{i}(x)(\gamma(d x)-\lambda(d x)) F_{j} \int_{B(R)} g_{j}(x)(\gamma(d x)-\lambda(d x))\right] \\
& +\mathbb{E}\left[\sum_{i=1}^{n} F_{i}^{2}\left(\int_{B(R)} g_{i}(x)(\gamma(d x)-\lambda(d x))\right)^{2}\right] \\
= & \mathbb{E}\left[\sum_{i=1}^{n} F_{i}^{2} \int_{B(R)} g_{i}^{2}(x) \lambda(d x)\right] \\
= & \mathbb{E}\left[\int_{B(R)} u^{2}(x) \lambda(d x)\right],
\end{aligned}
$$

which shows that (2.11) extends to the closure of $\mathcal{P}_{0}$ in $L^{2}(\Omega \times B(R))$ by density and a Cauchy sequence argument.

\section{Acknowledgements}

The author would like to thank an anonymous referee for useful comments.

\section{References}

G. Acosta and R. G. Durán. Divergence operator and related inequalities. SpringerBriefs in Mathematics. Springer, New York (2017). ISBN 978-1-4939-6983-8; 978-1-4939-6985-2. MR3618122.

S. Albeverio, Yu. G. Kondratiev and M. Röckner. Analysis and geometry on configuration spaces. J. Funct. Anal. 154 (2), 444-500 (1998). MR1612725.

A. D. Barbour. Asymptotic expansions based on smooth functions in the central limit theorem. Probab. Theory Relat. Fields 72 (2), 289-303 (1986). MR836279. 
S. Bourguin and G. Peccati. The Malliavin-Stein method on the Poisson space. In Stochastic analysis for Poisson point processes, volume 7 of Bocconi Springer Ser., pages 185-228. Bocconi Univ. Press (2016). MR3585401.

S. Campese. Optimal convergence rates and one-term edge-worth expansions for multidimensional functionals of Gaussian fields. ALEA Lat. Am. J. Probab. Math. Stat. 10 (2), 881-919 (2013). MR3149458.

L. H. Y. Chen, L. Goldstein and Q.-M. Shao. Normal approximation by Stein's method. Probability and its Applications (New York). Springer, Heidelberg (2011). ISBN 978-3-642-15006-7. MR2732624.

M. Clausel, J.-F. Coeurjolly and J. Lelong. Stein estimation of the intensity of a spatial homogeneous Poisson point process. Ann. Appl. Probab. 26 (3), 14951534 (2016). MR3513597.

C. Döbler and G. Peccati. The fourth moment theorem on the Poisson space. Ann. Probab. 46 (4), 1878-1916 (2018). MR3813981.

C. Döbler, A. Vidotto and G. Zheng. Fourth moment theorems on the Poisson space in any dimension. Electron. J. Probab. 23, Paper No. 36, 27 (2018). MR3798246.

G. Last. Stochastic analysis for Poisson processes. In Stochastic analysis for Poisson point processes, volume 7 of Bocconi Springer Ser., pages 1-36. Bocconi Univ. Press (2016). MR3585396.

I. Nourdin and G. Peccati. Stein's method and exact Berry-Esseen asymptotics for functionals of Gaussian fields. Ann. Probab. 37 (6), 2231-2261 (2009a). MR2573557.

I. Nourdin and G. Peccati. Stein's method on Wiener chaos. Probab. Theory Related Fields 145 (1-2), 75-118 (2009b). MR2520122.

I. Nourdin and G. Peccati. Cumulants on the Wiener space. J. Funct. Anal. 258 (11), 3775-3791 (2010). MR2606872.

D. Nualart and G. Peccati. Central limit theorems for sequences of multiple stochastic integrals. Ann. Probab. 33 (1), 177-193 (2005). MR2118863.

G. Peccati, J. L. Solé, M. S. Taqqu and F. Utzet. Stein's method and normal approximation of Poisson functionals. Ann. Probab. 38 (2), 443-478 (2010). MR2642882.

J.-J. Prat and N. Privault. Explicit stochastic analysis of Brownian motion and point measures on Riemannian manifolds. J. Funct. Anal. 167 (1), 201-242 (1999). MR1710625.

N. Privault. Connections and curvature in the Riemannian geometry of configuration spaces. J. Funct. Anal. 185 (2), 367-403 (2001). MR1856271.

N. Privault. Third cumulant Stein approximation for Poisson stochastic integrals (2018+). To appear in Journal of Theoretical Probability.

N. Privault and G. L. Torrisi. Density estimation of functionals of spatial point processes with application to wireless networks. SIAM J. Math. Anal. 43 (3), 1311-1344 (2011). MR2821586. 